Original Paper http://ajol.info/index.php/ijbcs http://indexmedicus.afro.who.int

\title{
Effet de quelques légumineuses fourragères sur la reproduction, la croissance et la carcasse chez les cochons d'inde (Cavia porcellus $\mathbf{L}$.)
}

\author{
J. Gwladys Kontchiachou NKANA ${ }^{1}$, Nathalie Ngouopo MWEUGANG ${ }^{3}$, \\ Soh Bah GERMANUS ${ }^{1}$, Yam Alphonsius SEMI ${ }^{1}$, Paulette NTSAFACK ${ }^{2}$, \\ Chistian AWANTU ${ }^{1}$, Christophe Temoa WANGBA ${ }^{1}$, Marie Noel Bertine NOUMBISSI ${ }^{2}$, \\ France Gina Tobou DJOUMESSI ${ }^{2}$ et Emile MIÉGOUÉ ${ }^{2 *}$
}

\author{
${ }^{I}$ Institut de Recherche Agronomique pour le développement (IRAD) Mankon-Bamenda PO. Box : 125 \\ Bamenda, Cameroun. \\ ${ }^{2}$ Université de Dschang, Faculté d'Agronomie et des Sciences Agricoles, Département des Productions \\ Animales, Laboratoire de Nutrition et Alimentation Animales. PO. Box: 188 Dschang, Cameroun. \\ ${ }^{3}$ Université de Ngaoundéré, Faculté des Sciences, Département des Sciences biologiques, Laboratoire de \\ Zoologie Appliquée. PO. Box: 454 Ngaoundéré, Cameroun. \\ *Corresponding author; E-mail: emile.miegoue@univ-dschang.org /migoumile@yahoo.fr;
} Tél: (+237) 697121297.

\section{RESUME}

Malgré l'importance de la caviaculture en milieu rural, la maîtrise de l'alimentation des cochons d'inde reste encore un handicap à cet élevage. C'est ainsi qu'entre octobre 2018 et mars 2019, un essai a été mené à la Ferme d'Application et de Recherche (FAR) de l'Université de Dschang en vue d'évaluer l'effet de quelques légumineuses fourragères comme source de protéine dans la ration sur les performances de production du cochon d'Inde. Pour cet essai, 56 cochons d'Inde de 5 mois d'âge de race locale pesant en moyenne $450 \pm 50 \mathrm{~g}$ nés à la FAR ont été utilisés. En effet, 48 femelles repartis en quatre lots ont été mises en croisement avec 2 mâles par lot pendant une période de 31 jours selon un dispositif factoriel. Les animaux de chaque lot recevaient la graminée ad libitum associé à $20 \mathrm{~g} / \mathrm{j} /$ animal de l'aliment composé contenant ou non une légumineuse. Les résultats montrent que les tailles de la portée sont restées comparables entre les traitements. Les taux de mortalité pré et post-sevrage $(15,38 \%$ et $9,10 \%)$ ainsi que le poids des cochonnets à 8 semaines les plus élevés ont été enregistrés avec la ration contenant Arachis glabrata. A la naissance et au sevrage, le poids moyen le plus élevé des cochonnets a été obtenu avec la ration contenant Desmodium intortum. Les gains de poids des cochonnets sont restés comparables entre les traitements. Les rations contenant les légumineuses ont donné des rendements carcasses, le poids du foie et celui du cæcum significativement plus élevé. Compte tenu du coût de production, les légumineuses de cette étude peuvent être utilisées comme des sources de protéine de choix pour l'amélioration des performances de production des cobayes en milieu paysan.

(C) 2020 International Formulae Group. All rights reserved.

Mots clés : Arachis glabrata, Calliandra calothyrsus, Desmodium intortum, Panicum maximum, Performances de production. 


\title{
Effect of some legumes forages on reproduction, growth and carcass of Guinea pigs (Cavia porcellus L.)
}

\begin{abstract}
Despite the importance of caviaculture in rural landscape, cavie's feeding remains a handicap for this breeding. Thus, between October 2018 and March 2019, experiments were conducted at the Teaching and Experimental Farm of University of Dschang on the evaluation of some leguminaceous plants as protein source in the diet on production performances of Guinea pig. A total of 56 adults Guinea pigs of about 5 months and $450 \pm 50 \mathrm{~g}$ were used for the experiments. Females were first put in breeding with 8 males for a period of 31 days and then organized in a completely randomized design of four groups of 12 females each. Each group received Panicum maximum ad libitum and $20 \mathrm{~g} \mathrm{DM}$ /day/animal of a diet corresponding to the group. Mains results of this study showed that no significant difference $(\mathrm{P}>0.05)$ has been noticed for mean litter size. Total mortality rate was 8.33 for diet containing Calliandra calothyrsus. No mortality was registered for others groups. High pre-weaning and post-weaning mortality (15.38 and 9.10\%) was obtained with treatment based on Arachis glabrata. From birth to $8^{\text {th }}$ week of age, no significant difference $(\mathrm{P}>0.05)$ was observed between average live weights. Total and daily weight gains remain comparable $(\mathrm{P}>0.05)$ in weaning and in post-weaning whatever the diet. Results for carcass evaluation showed that diet containing these plants as sources of protein has permitted to obtain the highest values. The same result was observed with the liver weight and cæcum. Considering the low production cost, legumes plants appear to be good protein sources for the improvement of production performances of the guinea pigs in small farmer environment.
\end{abstract}

(C) 2020 International Formulae Group. All rights reserved.

Keywords : Arachis glabrata, Calliandra calothyrsus, Desmodium intortum, Panicum maximum, production performances.

\section{INTRODUCTION}

Dans les tropiques, le principal handicap à la production des herbivores domestiques réside surtout dans la valeur nutritive des fourrages utilisés (Miégoué et al., 2018a). En effet, la qualité des fourrages n'est bonne qu'en début de saison des pluies et, se détériore au fur et à mesure que la saison avance (Pamo et al., 2007). Cette détérioration de la qualité fourragère s'accompagne d'une baisse de la digestibilité. Les animaux qui ne consomment que les graminées pendant la mauvaise saison ont des difficultés à extérioriser leurs potentialités de production (Miégoué et al., 2018b).

Plusieurs solutions ont été envisagées dans le sens d'améliorer la qualité nutritive des graminées fourragères. L'une des solutions la plus facilement réalisable est l'utilisation des légumineuses fourragères dont la valeur nutritive est établie comme complément d'aliment pauvres (Miegoué et al., 2019a ; Boukila et al., 2009). L'utilisation des légumineuses peut constituer également une solution efficace dans la réduction du coût de production des herbivores domestiques en général et du cochon d'inde en particulier.

Par ailleurs, selon Miégoué et al. (2019b), pour minimiser les coûts liés à l'alimentation des cochons d'Inde d'élevage, il faut maximiser l'ingestion de fourrages disponibles et procéder à une distribution rationnée de concentrés et de compléments minéraux vitaminés (CMV). Quelques légumineuses fourragères dont Desmodium intortum, Calliandra calothyrsus, Leucaena leucocephala et Arachis glabrata sont réputées pour un excellent potentiel de croissance en Afrique tropical (Miégoué et al., 2019b). Leurs feuilles et celles des autres plantes, riches en protéines, peuvent donc être utilisées comme supplément alimentaire pour les cochons d'Inde. C'est ainsi que Desmodium intortum ou Arachis glabrata ont été utilisés dans l'alimentation des cochons d'Inde avec de bons 
résultats (Kenfack et al., 2006 ; Miégoué et al., 2018b).

Par ailleurs, en Afrique en général et au Cameroun en particulier, plusieurs espèces végétales possèdent un potentiel fourrager certain mais, non encore exploré en caviaculture (Miégoué et al., 2018c). La présente étude se propose de manière générale de contribuer à l'amélioration de l'alimentation des cochons d'Inde à travers la valorisation des ressources locales disponible à moindre coût.

\section{MATERIEL ET METHODES}

\section{Site expérimental}

L'étude s'est déroulée d'octobre 2018 à mars 2019 à la Ferme d'Application et de Recherche (FAR) de l'Université de Dschang, située entre $5^{\circ} 25^{\prime}$ et $5^{\circ} 30^{\prime}$ de Latitude Nord, $10^{\circ} 0^{\prime}$ et $10^{\circ} 5^{\prime}$ de Longitude Est et à une altitude d'environ $1420 \mathrm{~m}$ dans l'Ouest Cameroun. Le climat de la région est équatorial de type camerounien d'altitude, avec une température moyenne annuelle de $20{ }^{\circ} \mathrm{C}$. $\mathrm{La}$ saison sèche va de mi-novembre à mi-mars, la saison des pluies de mi-mars à mi-novembre et correspond à la principale période des cultures. Les précipitations moyennes annuelles varient entre 1500 et $2000 \mathrm{~mm}$, avec une humidité relative oscillant entre $40 \%$ (en pleine saison sèche) et $97 \%$ (durant les grandes pluies).

\section{Matériel animal et logement}

Un total de 56 cobayes adultes dont 48 femelles et 8 mâles âgés de 5 mois et pesant $450 \pm 50 \mathrm{~g}$ a été utilisé. Tous les animaux utilisés étaient achetés dans les villages environnants et élevés à la Ferme. Pour parer à une déficience éventuelle en vitamine $\mathrm{C}$, cette dernière était distribuée quotidiennement à tous les animaux via l'eau de boisson (01 comprimé de $240 \mathrm{mg}$ dans 1,5 litre d'eau) servie à volonté.

Les animaux ont été répartis dans des loges faites de contreplaqués, mesurant chacune $1 \mathrm{~m}$ de longueur, $0,8 \mathrm{~m}$ de largeur et $0,6 \mathrm{~m}$ de hauteur et, munis d'un dispositif d'éclairage servant aussi de système de chauffage. Les animaux ont été élevés au sol, sur une litière en copeaux de bois sec non traité de $5 \mathrm{~cm}$ d'épaisseur, renouvelée tous les 2 jours pour éviter l'accumulation des fèces et des urines. Chaque compartiment ou loge était équipé de 2 mangeoires en bois pour l'aliment concentré et de 2 abreuvoirs en béton.

\section{Matériel végétal}

Le matériel végétal était constitué de Panicum maximum et des feuilles de légumineuses (Arachis glabrata, Desmodium intortum ou Calliandra colothyrsus). Les graminées étaient fauchées dans le champ fourragé de la ferme la veille et pré-fané dans l'un des logis du bâtiment d'élevage avant d'être directement servis le lendemain aux animaux. Les feuilles de légumineuses, quant à elles, ont été récoltées avant floraison, séchées, broyées et incorporées dans un aliment composé.

\section{Conduite des essais \\ Préparation des différentes rations}

Les ingrédients ont été achetés chez les revendeurs des sous-produits agricoles de la ville de Dschang. Les proportions des différents ingrédients utilisés dans la fabrication de la provende de même que la valeur nutritive de celle-ci sont consignées dans le Tableau 1. En effet, un échantillon de $100 \mathrm{~g}$ de chacune des rations expérimentales et des différents fourrages a été prélevé, séché, broyé à l'aide d'une broyeuse artisanale trimarteau munis d'un tamis de $1 \mathrm{~mm}$ de maille et conservé dans les sachets en plastique pour l'évaluation de leur teneur en matière sèche (MS), en matière organique (MO), en protéine brute $(\mathrm{PB})$ et en cellulose brute (CB) tel que décrite par AOAC (1990)

Les différentes rations ainsi préparées ont servi à la fabrication des granulés à l'aide d'une granuleuse mécanique de fabrication artisanale. 
La teneur en minéraux a été évaluée par la méthode décrite par Pauwel et al. (1992). La composition chimique des différents fourrages est présentée dans le Tableau 2.

\section{Evaluation des performances de reproduction et de croissance en fonction des différentes rations}

Les animaux étaient mis en croisement avec un ratio de 2 mâles pour 12 femelles par lot pendant 31 jours, et ensuite, les mâles ont été isolés (Pamo et al., 2005). Les animaux ont été identifiés par des boucles numérotées fixées à l'oreille gauche. Chacune des rations était distribuée deux fois par jour entre $8 \mathrm{~h}-9 \mathrm{~h}$ et $16 \mathrm{~h}$ - 18h respectivement aux lots concernés ainsi :

PM: P. maximum ad-libitum $+20 \mathrm{~g} \mathrm{de}$ R0; PM-AG: P. maximum ad-libitum $+20 \mathrm{~g}$ de R1; PM-CC: P. maximum ad-libitum $+20 \mathrm{~g}$ de R2, PM-DI: P. maximum ad-libitum $+20 \mathrm{~g}$ de R3

Les lots homogènes (sexe, poids et âge) de poids moyen $450 \pm 50 \mathrm{~g}$ ont été constitués à partir des différents traitements.

Les femelles gestantes et les femelles allaitantes recevaient la même quantité d'aliment jusqu'au sevrage des petits ( 21 jours après leur naissance). Aux sevrons, les quantités d'aliments servies ont été ajustées en fonction de leur poids à partir des quantités servies aux mères.

\section{Performances de reproduction}

Dès le début de l'expérience, l'effectif des femelles mises en reproduction a été enregistré. A la mise-bas, l'effectif des femelles ayant mise-bas ainsi que la date de mise-bas de chaque femelle ont été notés. La date de naissance de chaque nouveau-né vivant ainsi que le nombre de cochonnets mort-nés et nés vivants ont également été notés, de même que d'éventuels avortements. Ensuite, le nombre de cochonnets morts avant sevrage et le nombre de cochonnets sevrés ont été enregistrés. Après le sevrage, le nombre de jeunes post-sevrés vivants à 8 semaines d'âge a également été enregistré.

Performances de croissance

Chaque nouveau-né était identifié à la naissance et les pesés ont ensuite été faites toutes les semaines jusqu'à la $3^{\text {ème }}$ semaine (sevrage). Les poids enregistrés ont permis d'évaluer la croissance pré-sevrage des cochonnets. Au sevrage, chaque cochonnet a été sexé et envoyé dans le lot d'engraissement correspondant au régime alimentaire de sa mère. Leurs poids ont également été pris toutes les semaines jusqu'à leur $8^{\text {ème }}$ semaine et ceci nous a permis d'évaluer les gains de poids (gain totaux (GT) et gain moyen quotidien (GMQ)) à la $8^{\text {ème }}$ semaine.

\section{Evaluation des caractéristiques de la carcasse et des organes du tube digestif}

A la fin de l'essai d'alimentation, 5 animaux par traitement âgés de 8 semaines, pris au hasard et mis à jeun pendant $12 \mathrm{~h}$, ont été abattus par dislocation cervicale puis saignés à la gorge pour l'évaluation des caractéristiques de la carcasse, les pesées et les mesures des organes. La carcasse (rendement carcasse) et la proportion des différents organes (tube digestif en entier, intestin, peau, foie et cæcum) par rapport au poids vif à l'abattage ont été évaluées. Toutes les pesées ont été faites à l'aide d'une balance digitale de capacité $3 \mathrm{~kg}$ et de précision $1 \mathrm{~g}$. La longueur de l'intestin a été mesurée à l'aide d'un mètre ruban de précision $1 \mathrm{~mm}$.

\section{Analyse des données}

Les données collectées ont été soumise à l'analyse de variance à un facteur (ration alimentaire) suivant le model linéaire général (MLG). Lorsque les différences significatives existaient entre les traitements, la séparation des moyennes était faite par le test de Waller Dunkan au seuil de signification 5\% (Steel et Torrie, 1980). Le logiciel d'analyse utilisé a été SPSS 19.0. 
Tableau 1: Composition en pourcentage des différents ingrédients et valeur nutritive des rations expérimentales.

\begin{tabular}{|c|c|c|c|c|}
\hline \multirow[t]{2}{*}{ ingrédients } & \multicolumn{3}{|c|}{ Rations } & \multirow[b]{2}{*}{ R3 } \\
\hline & R0 & $\mathbf{R 1}$ & $\mathbf{R 2}$ & \\
\hline Remoulage & 31 & 25 & 25,5 & 26 \\
\hline Maïs & 30 & 24 & 25 & 25 \\
\hline Tourteau de coton & 5 & 4 & 4 & 4 \\
\hline Tourteau de palmiste & 25 & 20 & 21 & 21 \\
\hline Tourteau de soja & 2 & 1,5 & 1,5 & 1,5 \\
\hline Farine de poisson & 3 & 2,5 & 2,5 & 2,5 \\
\hline Poudre de coquille & 2 & 1 & 1,5 & 1,5 \\
\hline Prémix* & 1 & 1 & 1 & 1 \\
\hline Sel de cuisine & 1 & 1 & 1 & 1 \\
\hline A. glabrata & 0 & 20 & 0 & 0 \\
\hline C. calothyrsus & 0 & 0 & 17 & 0 \\
\hline D. intortum & 0 & 0 & 0 & 16,5 \\
\hline TOTAL & 100 & 100 & 100 & 100 \\
\hline \multicolumn{5}{|c|}{ Valeur nutritive } \\
\hline Matière sèche (MS en \%) & 91,97 & 92,10 & 92,47 & 92,90 \\
\hline Matière organique (\%MS) & 89,83 & 90,81 & 87,29 & 89,78 \\
\hline Protéine brute (\%MS) & 15,76 & 16,23 & 16,36 & 16,07 \\
\hline Lipides (\%MS) & 08,74 & 04,80 & 06,65 & 06,32 \\
\hline Cellulose brute (\%MS) & 17,48 & 19,94 & 12,95 & 15,44 \\
\hline Cendre (\%MS) & 10,17 & 09,19 & 12,71 & 10,22 \\
\hline $\mathrm{EM}(\mathrm{Kcal} / \mathrm{Kg} \mathrm{MS})$ & 2576,5 & 2471,56 & 2533,85 & 2549,55 \\
\hline
\end{tabular}

Tableau 2: Composition chimique des fourrages.

\begin{tabular}{lllll}
\hline Composition chimique & \multicolumn{4}{c}{ Différents fourrages } \\
\cline { 2 - 5 } & $\begin{array}{l}\text { Panicum } \\
\text { maximum }\end{array}$ & $\begin{array}{l}\text { Arachis } \\
\text { glabrata }\end{array}$ & $\begin{array}{l}\text { Calliandra } \\
\text { calothyrsus }\end{array}$ & $\begin{array}{l}\text { Desmodium } \\
\text { intortum }\end{array}$ \\
\hline Matière sèche (\%) & 91,76 & 90,92 & 93,29 & 92,38 \\
Matière Organique (\%MS) & 85,88 & 88,02 & 90,22 & 89,65 \\
Protéine Brute (\%MS) & 13,45 & 20,00 & 23,98 & 23,79 \\
Lipides (\%MS) & 2,67 & 2,95 & 5,58 & 3,50 \\
Cellulose brute (\%MS) & 33,0 & 26,30 & 31,63 & 29,63 \\
Cendres (\%MS) & 14,12 & 11,98 & 9,78 & 10,35 \\
\hline & Teneurs en minéraux (mg/kg MS) \\
\hline Phosphore & 1973,25 & 1434,75 & 1704,00 & 1973,20 \\
Calcium & 3778,80 & 3438,80 & 2998,80 & 3798,80 \\
Magnésium & 1329,20 & 964,70 & 2422,7 & 2908,70 \\
Potassium & 162,50 & 162,50 & 162,5 & 818,75 \\
Sodium & 43,92 & 24,40 & 20,20 & 10,42 \\
Rapport Ca/P & 1,92 & 2,40 & 1,76 & 1,93 \\
\hline
\end{tabular}




\section{RESULTATS \\ Performances de reproduction chez les femelles mises en reproduction}

L'inclusion de la poudre des feuilles de légumineuses dans les rations n'a eu aucune influence significative $(P>0,05)$ sur les tailles de la portée (Tableau 3). Le taux de fertilité a peu varié en fonction des rations. Le traitement PM-DI a permis d'obtenir le taux de fertilité le plus élevé $(91,67 \%)$. La ration témoin (PM) a donné le taux de fertilité le plus faible $(66,67 \%)$. Les traitements PM et PM-CC ont permis d'obtenir un taux de fécondité de $100 \%$ et, PM-DI 125\%. L'ajout des légumineuses dans les rations a contribué à réduire les mortalités des cochonnets à la naissance. Le taux le plus élevé (8,33\%) a été obtenu avec les animaux soumis à la ration PM-CC et, aucune mortalité n'a été observée pour les autres lots.

Pour ce qui est des mortalités pré et post-sevrage, les taux les plus élevés $(15,38 \%$ et $09,10 \%$ ) étaient obtenues avec le traitement PM-AG. Pendant la période pré-sevrage, les mortalités sont restées faibles pour les traitements PM $(8,33 \%)$ et PM-CC $(9,10 \%)$ et nulles pour le traitement PM-DI. Pendant la période post-sevrage, aucune mortalité n'a été observée avec les traitements PM, PM-CC et PM-DI.

Le rapport entre le nombre de cochonnets nés vivants et le nombre de cochonnets nés ou viabilité à la naissance de $100 \%$, a été obtenue chez les animaux soumis aux rations PM, PM-AG et PM-DI et, le traitement PM-CC a donné un taux de 91,67\%. Par contre, la viabilité au sevrage ou taux de sevrage qui est le nombre de cochonnets sevrés par rapport au nombre de cochonnets nés vivants a été plus faible $(84,62 \%)$ avec le traitement PM-AG et plus élevé $(100 \%)$ avec PM-DI, suivit du traitement PM $(91,67 \%)$ et PM-CC $(90,91 \%)$.

Le taux de survie des jeunes était de $100 \%$ chez les animaux nourris aux rations PM, PM-CC et PM-DI et, les cochonnets nourris à la ration contenant $A$. glabrata ont présenté la viabilité la plus faible $(90,91 \%)$.

\section{Performances de croissance des cochons d'Inde en fonction des rations}

L'inclusion de la poudre des feuilles de légumineuses n'a eu aucun effet significatif ( $P$ $>0,05)$ sur le poids des animaux à la naissance ou au sevrage (Tableau 4). Il en a été de même pour les gains totaux et les gains moyens quotidiens.

L'inclusion de la poudre des feuilles de légumineuses n'a eu aucun effet significatif (P $>0,05)$ sur les gains de poids ni au sevrage ni à 8 semaines d'âge.

Effet de l'inclusion de la poudre des feuilles de légumineuses dans la ration sur les caractéristiques de la carcasse

En dehors du poids du cœur, des rendements carcasses et de la proportion de la tête (Tableau 5), tous les autres paramètres à l'abattage ont significativement $(\mathrm{P}<0,05)$ été affectés par la variation des différentes sources de protéine de la ration. En effet, le poids vif à l'abattage, les poids des organes et des carcasses significativement $(\mathrm{P}<0,05)$ les plus élevés ont été obtenus avec les animaux soumis à la ration contenant $D$. intortum et les plus faibles avec les animaux soumis à la ration témoin.

Le poids vif à l'abatage, le poids de la tête, du tube digestif et les poids carcasses pour les traitements PM-AG, PM-CC et PM-DI ont été comparables $(\mathrm{P}>0,05)$ mais significativement supérieures à ceux des animaux du lot témoin. En ce qui concerne le poids du cœur, aucune différence significative $(\mathrm{P}>0,05)$ n'a été observée entre les traitements. L'inclusion de la poudre des feuilles de légumineuses dans la ration n'a eu aucun effet significatif $(P>0,05)$ sur les rendements carcasse.

L'inclusion de la poudre des feuilles de légumineuses a induit des effets significatifs $(\mathrm{P}$ $<0,05)$ sur les proportions du foie et du tube digestif par rapport au poids vif du cobaye. Les valeurs significativement $(\mathrm{P}<0,05)$ les plus élevées ont été obtenues chez les animaux de la ration PM et les plus faibles chez ceux de la ration PM-AG pour le tube digestif. Les traitements PM-CC et PM-DI ont donné le rapport du tube digestif comparable entre eux, 
et à celui des animaux nourris à la ration PM d'une part et à celui des animaux soumis à la ration PM-AG d'autre part. Les animaux nourris à la ration PM-DI ont eu la proportion $\mathrm{du}$ foie la plus élevée mais, statistiquement comparable $(\mathrm{P}>0,05)$ à celles des traitements PM-AG et PM-CC. Par contre, les animaux de la ration PM ont donné des proportions significativement les plus faibles que celui des autres traitements.

\section{Effet de la complémentation sur les caractéristiques de quelques organes de digestion chez les cochons d'Inde}

Les différentes rations ont significativement $(\mathrm{P}<0,05)$ influencé les caractéristiques des organes de la digestion en dehors de la longueur et la densité de l'intestin grêle (Tableau 6).

Les poids des foies des animaux nourris aux rations $\mathrm{PM}-\mathrm{AG}, \mathrm{PM}-\mathrm{CC}$ et PM-DI ont été comparables $(\mathrm{P}>0,05)$ entre eux et, significativement supérieures $(\mathrm{P}<0,05)$ à ceux des animaux soumis à la ration PM. Les animaux nourris à la ration PM-DI ont eu des poids $\mathrm{du}$ gros intestin significativement supérieurs à ceux des animaux soumis aux traitements PM, PM-AG, et PM-CC. Ces trois derniers traitements ont donné des poids du gros intestin comparables $(\mathrm{P}>0,05)$ entre eux et significativement $(P<0,05)$ plus petits que celui du traitement PM-DI. Le traitement PM$\mathrm{CC}$ a permis d'obtenir le poids moyen de l'intestin grêle statistiquement comparable $(\mathrm{P}>$
$0,05)$ à celui des traitements PM-AG et PM-DI d'une part, et d'autre part, à celui du traitement PM. Le poids du cæcum, significativement ( $\mathrm{P}$ $<0,05)$ le plus élevé, a été obtenu avec le traitement PM-DI et le plus faible avec le traitement PM-CC. Le traitement PM-AG a produit la longueur $\mathrm{du}$ gros intestin significativement $(\mathrm{P}<0,05)$ le plus élevé mais, cette valeur est restée comparable $(\mathrm{P}>0,05)$ à celles obtenues avec les traitements PM-CC et PM-DI. Le traitement PM, par ailleurs, a permis d'obtenir la longueur du gros intestin significativement $(\mathrm{P}<0,05)$ la plus faible. La longueur et la densité de l'intestin grêle chez les animaux nourris avec $P$. maximum n'ont pas été significativement $(\mathrm{P}>0,05)$ influencées par l'inclusion de la poudre des feuilles de légumineuses.

La longueur du cæcum a significativement $(P>0,05)$ été influencée par les différentes rations. $\mathrm{La}$ valeur significativement $(P<0,05)$ la plus élevée a été obtenue avec le traitement contenant $C$. calothyrsus et la plus faible avec le traitement témoin. Les rations contenant $D$. intortum ont permis d'obtenir la longueur du cæcum significativement supérieure $(\mathrm{P}<0,05)$ à celle des rations témoins mais, comparable $(\mathrm{P}>$ $0,05)$ à celle du traitement contenant $A$. glabrata. Ce dernier traitement, quant à lui, a donné une longueur du cæcum statistiquement comparable $(\mathrm{P}>0,05)$ à celle des animaux du traitement témoin.

Tableau 3: Performances moyennes de reproduction chez les cochons d'Inde en fonction des différentes rations.

\begin{tabular}{lcccc}
\hline \multirow{2}{*}{ Paramètres } & \multicolumn{4}{c}{ Traitements } \\
\cline { 2 - 5 } & PM & PM-AG & PM-CC & PM-DI \\
\hline Taux de fertilité (\%) & 66,67 & 83,33 & 75,00 & 91,67 \\
Taille de la portée* & $1,50 \pm 0,53$ & $1,30 \pm 0,48$ & $1,38 \pm 0,51$ & $1,36 \pm 0,50$ \\
Taux de fécondité (\%) & 100 & 108 & 100 & 125 \\
Taux de fécondité nette (\%) & 100 & 108 & 91,67 & 125 \\
Mortalité à la naissance (\%) & 0 & 0 & 8,33 & 0 \\
Mortalité au sevrage (\%) & 8,33 & 15,38 & 9,09 & 0 \\
Mortalité post-sevrage (\%) & 0 & 9,09 & 0 & 0 \\
\hline *: Aucune différence significative $(\mathrm{P}>0,05)$ n'a été observée pour ce paramètre quelle que soit la ration.
\end{tabular}


Tableau 4: Gains totaux et gains moyens quotidiens des cochonnets de la naissance au sevrage, en fonction des rations alimentaires.

\begin{tabular}{|c|c|c|c|c|c|c|c|}
\hline \multirow{2}{*}{ Paramètres } & & \multicolumn{4}{|c|}{ Traitements } & \multirow[t]{2}{*}{$E S M$} & \multirow[t]{2}{*}{$\mathbf{P}$} \\
\hline & & PM & PM-AG & PM-CC & PM-DI & & \\
\hline Poids à la naissance (g) & đ우 & $78,83^{\mathrm{a}}(\mathbf{1 2})$ & $73,77^{\mathrm{a}}(\mathbf{1 3})$ & $73,09^{\mathrm{a}}(\mathbf{1 1})$ & $84,85^{\mathrm{a}}(\mathbf{1 5})$ & 2,31 & 0,219 \\
\hline Poids au sevrage (g) & ํㅜ & $166,09^{\mathrm{a}}(\mathbf{1 1})$ & $167,64^{\mathrm{a}}(\mathbf{1 1})$ & $159,60^{\mathrm{a}}(\mathbf{1 0})$ & $170,67^{\mathrm{a}}(\mathbf{1 5})$ & 3,70 & 0,773 \\
\hline Poids à 8 semaines (g) & ơt & $319,36^{\mathrm{a}}(\mathbf{1 1})$ & $332,30^{\mathrm{a}}(\mathbf{1 0})$ & $320,40^{\mathrm{a}}(\mathbf{1 0})$ & $316,53^{\mathrm{a}}(\mathbf{1 5})$ & 3,67 & 0,371 \\
\hline G T au sevrage(g) & $\widehat{\jmath} q$ & $86,27^{\mathrm{a}}$ & $88,73^{\mathrm{a}}$ & $84,70^{\mathrm{a}}$ & $85,80^{\mathrm{a}}$ & 1,89 & 0,912 \\
\hline G T Post-sevrage & $\widehat{c}$ o우 & $118,82^{\mathrm{a}}$ & $123,67^{\mathrm{a}}$ & $125,44^{\mathrm{a}}$ & $116,60^{\mathrm{a}}$ & 1,56 & 0,148 \\
\hline GMQ (g/j) & 후우 & $4,11^{\mathrm{a}}$ & $4,23^{\mathrm{a}}$ & $4,03^{\mathrm{a}}$ & $4,09^{\mathbf{a}}$ & 0,09 & 0,912 \\
\hline GMQ (g/j) Post-sevrage & §o우 & $3,39^{\mathrm{a}}$ & $3,53^{\mathrm{a}}$ & $3,58^{\mathrm{a}}$ & $3,33^{\mathrm{a}}$ & 0,045 & 0,148 \\
\hline
\end{tabular}

Tableau 5: Caractéristiques de la carcasse des cochons d'Inde nourris en fonction des différentes rations.

\begin{tabular}{|c|c|c|c|c|c|c|}
\hline \multirow[t]{2}{*}{ Paramètres } & \multicolumn{4}{|c|}{ Traitements } & \multirow{2}{*}{ ESM } & \multirow{2}{*}{$\mathbf{P}$} \\
\hline & PM & PM-AG & PM-CC & PM-DI & & \\
\hline \multicolumn{7}{|l|}{ Poids (g) } \\
\hline PVa & $291,40^{\mathrm{b}}$ & $388,20^{\mathrm{a}}$ & $423,80^{\mathrm{a}}$ & $426,60^{\mathrm{a}}$ & 15,54 & 0,001 \\
\hline Tête & $42,80^{\mathrm{b}}$ & $55,60^{\mathrm{a}}$ & $52,40^{\mathrm{a}}$ & $53,00^{\mathrm{a}}$ & 1,39 & 0,001 \\
\hline Cœur & $1,00^{\mathrm{a}}$ & $1,80^{\mathrm{a}}$ & $1,80^{\mathrm{a}}$ & $1,60^{\mathrm{a}}$ & 0,19 & 0,393 \\
\hline Tube digestif & $88,00^{\mathrm{b}}$ & $115,80^{\mathrm{a}}$ & $106,40^{\mathrm{a}}$ & $115,00^{\mathrm{a}}$ & 3,32 & 0,002 \\
\hline Carcasse commerciale & $122,40^{\mathrm{b}}$ & $199,60^{\mathrm{a}}$ & $175,20^{\mathrm{a}}$ & $213,80^{\mathrm{a}}$ & 11,28 & 0,009 \\
\hline Carcasse classique & $185,60^{\mathrm{b}}$ & $282,40^{\mathrm{a}}$ & $254,20^{\mathrm{a}}$ & $294,80^{\mathrm{a}}$ & 13,16 & 0,005 \\
\hline \multicolumn{7}{|l|}{ Rendement (\%) } \\
\hline Carcasse commerciale & $41,71^{\mathrm{a}}$ & $46,78^{\mathrm{a}}$ & $45,08^{\mathrm{a}}$ & $49,28^{\mathrm{a}}$ & 1,22 & 0,163 \\
\hline Carcasse classique & $63,49^{\mathrm{a}}$ & $66,19^{\mathrm{a}}$ & $65,44^{\mathrm{a}}$ & $68,86^{\mathrm{a}}$ & 1,02 & 0,327 \\
\hline \multicolumn{7}{|c|}{ Proportions des organes (\%) } \\
\hline Tête /PVa & $14,74^{\mathrm{a}}$ & $13,03^{\mathrm{a}}$ & $13,51^{\mathrm{a}}$ & $12,84^{\mathrm{a}}$ & 0,34 & 0,196 \\
\hline Foie /PVa & $2,18^{\mathrm{b}}$ & $3,10^{\mathrm{a}}$ & $3,23^{\mathrm{a}}$ & $3,32^{\mathrm{a}}$ & 0,14 & 0,006 \\
\hline Tube digestif /PVa & $30,15^{\mathrm{a}}$ & $27,18^{\mathrm{b}}$ & $27,42^{\mathrm{ab}}$ & $27,53^{\mathrm{ab}}$ & 0,49 & 0,059 \\
\hline
\end{tabular}


Tableau 6: Poids et longueurs de quelques organes de digestion chez les cochons d'Inde en fonction des traitements.

\begin{tabular}{|c|c|c|c|c|c|c|}
\hline \multirow{2}{*}{ Paramètres } & \multicolumn{4}{|c|}{ Traitements } & \multirow{2}{*}{$E S M$} & \multirow{2}{*}{$\mathbf{P}$} \\
\hline & $\overline{\mathrm{PM}}$ & PM-AG & PM-CC & PM-DI & & \\
\hline \multicolumn{7}{|l|}{ Poids (g) } \\
\hline Foie & $06,40^{\mathrm{b}}$ & $13,20^{\mathrm{a}}$ & $12,60^{\mathrm{a}}$ & $14,00^{\mathrm{a}}$ & 0,82 & 0,000 \\
\hline Gros intestin & $46,20^{\mathrm{b}}$ & $52,80^{\mathrm{b}}$ & $45,80^{\mathrm{b}}$ & $63,20^{\mathrm{a}}$ & 1,92 & 0,000 \\
\hline Intestin grêle & $11,00^{\mathrm{b}}$ & $14,60^{\mathrm{a}}$ & $12,60^{\mathrm{ab}}$ & $14,60^{\mathrm{a}}$ & 0,50 & 0,011 \\
\hline Cæcum & $34,20^{\mathrm{b}}$ & $38,00^{\mathrm{ab}}$ & $32,00^{\mathrm{b}}$ & $41,20^{\mathrm{a}}$ & 1,30 & 0,043 \\
\hline \multicolumn{7}{|c|}{ Longueur (cm) } \\
\hline Gros intestin & $92,40^{\mathrm{b}}$ & $108,70^{\mathrm{a}}$ & $111,10^{\mathrm{a}}$ & $106,40^{\mathrm{a}}$ & 6,95 & 0,000 \\
\hline Intestin grêle & $130,60^{\mathrm{a}}$ & $118,80^{\mathrm{a}}$ & $153,10^{\mathrm{a}}$ & $147,80^{\mathrm{a}}$ & 1,95 & 0,290 \\
\hline Cæcum & $11,20^{\mathrm{c}}$ & $12,60^{\mathrm{bc}}$ & $15,40^{\mathrm{a}}$ & $13,80^{\mathrm{ab}}$ & 0,51 & 0,013 \\
\hline
\end{tabular}

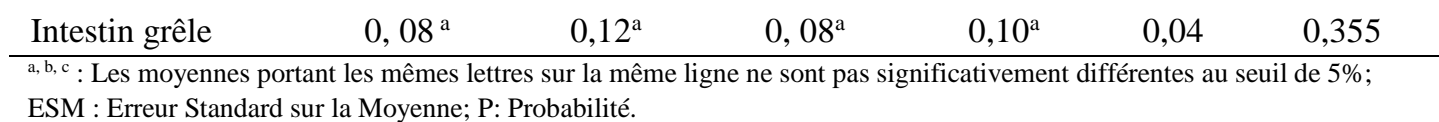

\section{DISCUSSION}

\section{Performances de reproduction}

Le taux de fertilité dans cette étude était compris entre 60 et $95 \%$. Les valeurs les plus élevées dans cet essai pourraient s'expliquer par le fait que les rations utilisées étaient des rations équilibrées et suffisamment riches en protéine pour permettre une bonne croissance fotale et par ailleurs, les poids moyens des femelles étaient suffisamment élevés pour supporter la gestation. Ces résultats concordent avec ceux de Kenfack et al. (2006) et Mweugang et al. (2016) qui présentent une augmentation du taux de fertilité avec le taux de protéine de la ration des cochons d'Inde pesant en moyenne $540 \mathrm{~g}$. Les taux de fécondité les plus élevés ont été obtenus avec les rations contenant les légumineuses.

La variation de la source d'azote n'a eu aucun effet significatif sur la taille de la portée. Ceci peut s'expliquer par le fait que les rations étaient iso-azotées et, les sources de protéines de la ration auraient donc été bien valorisées chez l'animal. Selon Kenfack et al. (2006), la taille de la portée peut être aussi liée au poids, à l'âge de la reproductrice et au numéro de mise-bas. Dans cette étude, les femelles mis en reproduction étaient primipares. Les valeurs obtenues dans le cadre de cette étude ont été inférieures à celles obtenues par Kouakou et al. (2012) chez des cochons d'Inde femelles supplémentés avec des granulés pour lapin tout au long de leur gestation. Les valeurs obtenues dans cette étude seraient liées au fait que les femelles utilisées étaient des primipares. En effet, les primipares engendrent généralement des portées faibles comparées à celles des multipares (Mètre, 2012).

Les mortalités à la naissance, au sevrage et après le sevrage ont été plus faibles chez les animaux recevant dans leurs rations des légumineuses dans le cadre de cette étude. Ces valeurs élevées peuvent être attribuées, entre autres à l'alimentation. En effet, chez les cochonnets, l'alimentation est un facteur de viabilité tant à la naissance qu'au sevrage. Les 
cochons d'Inde gestants malnutris présentent un taux élevé de mortinatalité, et la viabilité des petits au sevrage est très faible (Kenfack et al., 2006).

\section{Croissance pondérale des cochonnets pré- sevrés}

A la naissance, les cochonnets soumis aux rations contenant les légumineuses étaient plus lourds que ceux du lot témoin quelle que soit la graminée utilisée. Ceci peut être attribué à la qualité des protéines reçues par leurs mères. Les cochons d'Inde étant des herbivores, auraient mieux valorisé les protéines d'origine végétales. En effet, le cochon d'Inde est un herbivore monogastrique avec une forte capacité de consommation de fourrage (Dikko et al., 2009; Zougou et al., 2017a). Selon Niraj et Vardhan (2012), la consommation des aliments entièrement d'origine végétale chez les cochons d'Inde ralentit la digestion, permet une bonne absorption des aliments et un équilibre plus favorable des nutriments en fonction des calories liées à une bonne gestion de la croissance cellulaire. Un taux de protéines d'origine végétale de bonne qualité de $16 \%$ suffit à couvrir les besoins du cochon d'Inde à l'entretien. Ceci montre que les rations utilisées dans cet essai se sont montrées intéressantes pour les femelles gestantes.

$\mathrm{Au}$ sevrage, les poids des cochonnets dans tous les lots quelle que soit la graminée avaient doublé et même plus pour les animaux de certains lots. Ceci serait associé à la rapide croissance pré-sevrage caractéristique des cochons d'Inde. En effet, le poids vif du cochon d'Inde est plus que doublé lors du sevrage normal à 3 semaines et il doublera une nouvelle fois au cours des 6 semaines suivantes (Miégoué et al., 2018c). Ces résultats montrent que nos rations restent dans la marge des rations utilisables chez les cochons d'Inde. A 8 semaines, le poids moyen des animaux soumis à la ration témoin était comparable à ceux des animaux soumis aux autres rations. Ceci témoigne du fait que les rations utilisées dans cet essai avaient les mêmes niveaux de protéine et, les animaux ont ainsi valorisé les différentes rations de la même manière.
Dans la présente étude, que ce soit à la naissance, au sevrage ou à l'âge de 8 semaines, il a été constaté que les rations contenant les légumineuses avaient permis d'obtenir de meilleurs poids. Cette observation pourrait être attribuée aux faits que les cochons d'Inde sont des animaux herbivores et, ils valoriseraient mieux les protéines d'origine végétale. En effet, du fait du régime strictement herbivore, le besoin protéique du cochon d'Inde est modéré comparé à celui d'un carnivore : un taux de protéines d'origine végétale de bonne qualité de $16 \%$ suffit à couvrir les besoins du cobaye à l'entretien. Par ailleurs, le séchage et l'incorporation des différentes légumineuses dans l'aliment composé auraient atténué l'effet des facteurs antinutritionnels. Dans la présente étude, les gains moyens quotidiens (GMQ) les plus élevés ont été obtenus chez les animaux soumis à la ration $\mathrm{PM}-\mathrm{CC}(3,58 \mathrm{~g} / \mathrm{j})$. Cette valeur est supérieure à celle de $1,70 \mathrm{~g} / \mathrm{j}$ rapportée par Fonteh et al. (2005) chez les cochons d'Inde de 12 semaines.

\section{Caractéristiques de la carcasse et quelques organes impliqués dans la digestion}

Les différentes sources de protéine ont affecté significativement les caractéristiques de la carcasse chez le cochon d'Inde. Les poids vifs des animaux à l'abattage et les poids des différents types de carcasse étaient plus élevés chez les animaux nourris aux rations contenant les légumineuses par rapport aux témoins. Cette supériorité pondérale peut être attribuée à la qualité des protéines des rations contenant les légumineuses. En effet, selon Zougou et al. (2017b) les caractéristiques de la carcasse peuvent être améliorées par l'augmentation du taux et de la qualité de protéine dans la ration alimentaire.

Le rendement de la carcasse commerciale le plus élevé $(49,28 \%)$ obtenu chez les animaux du lot soumis aux rations contenant $D$. intortum dans cette étude est supérieur à la valeur $(42,4 \%)$ observée par Zougou et al. (2017b) chez les cochons d'Inde âgés de 23 semaines et pesant en moyenne 526 $\mathrm{g}$ à l'abattage. Cette différence serait due, entre autres à la différence de poids vif et d'âge à l'abattage des animaux. En effet, suivant la 
remarque faite par Liméa (2009), l'augmentation du poids à l'abattage améliore le rendement vrai de la carcasse.

Le poids du foie des cochons d'Inde des rations contenant les légumineuses était significativement supérieur à celui de la ration témoin. Ce Résultat serait probablement dû à une intense activité de cet organe qui intervient dans la détoxification des facteurs antinutritionnels contenus dans les différentes légumineuses. Le poids du foie le plus élevé observé chez les cochons d'Inde nourris à la ration PM-DI (11,60 g) est lié à la forte teneur en facteurs antinutritionnels du $D$. intortum de la ration. Tolera et Sundstøl (2000) ont dosé les facteurs antinutritionnels de la plante, et ont constaté qu'elle contient des niveaux importants de tanins $(45,4 \mathrm{mg} / 100 \mathrm{~g})$.

Les animaux du lot soumis à la ration contenant $D$. intortum ont présenté le poids du cæcum le plus élevé. Ceci pourrait être dû au fait qu'ils ont ingéré la plus grande quantité de fibre dans cette ration et les fibres favorisent le développement des organes impliqués dans la digestion. En effet, le cæcum chez le cochon d'Inde comme chez la plupart des pseudoruminants, est l'organe fortement impliqué dans la digestion, notamment de la cellulose. Le cæcum est, en effet, l'équivalent du rumen chez les ruminants (Lormeau, 2010). C'est le site privilégié de la digestion des fibres non dégradées par les enzymes, puisqu'il abrite la flore microbienne capable de digérer la cellulose (Picron, 2007). Plus l'aliment fournit des fibres, plus les micro-organismes sont sollicités et davantage se développe le cæcum qui les abrite.

\section{Conclusion}

$\mathrm{Au}$ terme de cette étude sur la croissance et les caractéristiques de la carcasse chez le cochon d'inde et dont l'objectif était de contribuer à l'amélioration de la production de cet animal, il ressort que les différents traitements ont permis d'améliorer les différents paramètres de la reproduction. En effets, les rations contenant les légumineuses ont permis d'obtenir de meilleurs taux de fertilité, de fécondité et de viabilité des cochonnets de la naissance à leur $8^{\text {ème }}$ semaine d'âge. Il en a été de même pour les poids des cochonnets de la naissance au sevrage. A la naissance et au sevrage, les gains totaux et les gains moyens quotidiens n'ont pas été influencés par la complémentation. Les différents traitements n'ont pas eu d'effet sur les poids des sevrons à la $8^{\text {ème }}$ semaine. Cependant, ils ont induit une amélioration significative des gains totaux et les gains moyens quotidiens à huit semaines d'âge. Par contre, la ration contenant $D$. intortum a relevé le niveau de ces gains. L'ajout des légumineuses dans les rations a également amélioré les rendements des carcasses. Par contre, le poids du foie n'a pas significativement été influencé par les différentes rations

Au regard des performances obtenues et l'influence des légumineuses de l'étude sur les coûts de production des cochons d'Inde, elles peuvent être utilisées en milieu paysan comme source de protéines pour améliorer les performances de production de ces animaux. Cependant, il serait préférable de caractériser les légumineuses utilisées afin de déterminer le niveau adéquat d'inclusion de chacune d'elle dans la ration du cochon d'Inde.

\section{CONFLIT D'INTERETS}

Les auteurs de cet article déclarent qu'il n'existe aucun conflit d'intérêt inhérent à sa publication.

\section{CONTRIBUTIONS DES AUTEURS}

NKJG et MNN: Conception et mise en place du dispositif expérimental; initiation du projet de manuscrit. GSB, SAY, NP, AC, WTC, NMNB et DTFG: Collecte des données et re-lecture du manuscrit. ME: Coordination de l'équipe de recherche.

\section{REMERCIEMENTS}

La réalisation de ce travail n'a été possible que grace à la participation de $\mathrm{Dr}$ Mube Hervé et les techniciens de laboratoire à qui nous adressons nos sincères remerciements

\section{REFERENCES}

A.O.A.C. (Association of Official Analytical Chemist). 1990. Official Method of 
Analysis $\quad\left(15^{\text {th }} \quad\right.$ edn $) . \quad$ A.O.A.C: Washington D.C.; $10 \mathrm{p}$.

Boukila B, Tendonkeng F, Pamo TE, Betfiang ME. 2009. Composition chimique et digestibilité in vitro de Desmodium uncinatum, Desmodium intortum et Arachis glabrata incubés seuls ou mélangés avec des chaumes de maïs. Livestock Research for Rural Development; 21(7).

Dikko AH, Egena SSA, Malik AA, Ibrahim H. 2009. Guinea Pig (Cavia Porcellus) as an Untapped Protein Source for Man: The Potentialities, Opportunities and Challenges. Proceeding of $14^{\text {th }}$ Annual Conference of Animal Science Association of Nigeria (ASAN). September $14^{\text {th }}-17^{\text {th }} 2009$, LAUTECH Ogbomoso, Nigeria.

Fonteh FA, Niba AT, Kudi AC, Tchoumboue J, Awah-Ndukum J. 2005. Influence of weaning age on the growth performance and survival of weaned guinea pigs. Livestock Research for Rural Development. 17(133). http://www.cipav.org.co/lrrd//rrd17/12/f ont17133.htm

Kenfack A, Tcoumboué J, Kamtchouing P, Ngoula F. 2006. Effets de la substitution par l'arachide fourragère (Arachis glabrata) de l'herbe à éléphant (Pennisetum purpureum) sur le nombre d'ovulations et les mortalités prénatales chez le cobaye (Cavia porcellus) adulte. Tropicultura, 24 (3): 143-156.

Kouakou N'GDV, Thys E, Danho M, Assidjo EN, Grongnet JF. 2012. Effet de Panicum maximum sur la productivité des femelles primipares durant le cycle de reproduction chez le cobaye (Cavia porcellus L.). Tropicultura, 30(1) : 24-36.

Limea T. 2009. Effets des conditions d'alimentation et d'abattage sur les caractéristiques de carcasse et de la viande du caprin créole. Thèse pour obtenir le grade de Docteur de l'Institut des Sciences et Industrie du Vivant et de l'environnement (Agro Paris Tech). 230p. Lormeau E. 2010. Contribution à l'étude de Cavia porcellus (Linné, 1758): atlas radiographique et ostéologique. Thèse d'exercice, Médecine Vétérinaire, Toulouse $\quad 3, \quad 207 \mathrm{p}$. http ://www.oatao.univ-toulouse.fr

Metre TK. 2012. Possibilités d'amélioration de l'élevage de cobaye (Cavia porcellus L.) au Sud Kivu, à l'est de la République Démocratique du Congo. Mémoire présente en u diplôme de Master complémentaire en gestion des ressources animales et végétales en milieux tropicaux. Université de Liège, Académie Universitaire Wollonie-Europe. 52p.

Miegoue E, Tendonkeng F, Ngouopo NM, Ntsafack P, Mouchili M, Pamo TE. 2018c. Reproductive Performance and Pre-Weaning Growth Performance of Guinea Pigs (Cavia Porcellus L.) Fed on Panicum Maximum or Pennisetum Purpureum. Int J Anim Sci., 2(6): 1037.

Miegoué E, Tendonkeng F, Ngouopo NM, Fossi J, Ntsafack P, Agwah ED, Fogang MD, Mouchili M, Pamo ET. 2018a. PostWeaning Growth Performance of Guinea Pigs (Cavia porcellus L.) Fed on Panicum maximum Supplemented with Graded Levels of Arachis glabrata in the Diet. Journal of Veterinary Science \& Animal Husbandry, 6(6): 601.

Miégoué E, Mweugang NN, Lemoufouet J, Tendonkeng F, Kouam KM, Pamo Tedonkeng E, Theodoropoulos G. 2019a. Variation in the level of caecal enterobacteria and lactobacilli in guinea pigs fed on graded level of Arachis glabrata associated to Panicum maximum or Pennisetum purpureum. Sustainable Development, Culture, Traditions Journal, Special Volume in Honor of Professor George I. Theodoropoulos, 4973. https://doi.org/

Miégoué E, Tendonkeng F, Mweugang Ngouopo N, Tatang MV, Ntsafack P, Lemoufouet J, Mouchili M, Pamo ET. 2019b. Ingestion and In vivo Digestibility of Pennisetum purpureum Supplemented with Graded Level of Arachis glabrata in Guinea Pigs (CaviaPorcellus). Austin Journal of Veterinary Science \& Animal Husbandry. 6(1): 1050. 
Miégoué E, Tendonkeng F, Mweugang NN, Lemoufouet J, Fossi J, Ntsafack P, Pamo TE. 2018b. Effect of Arachis glabrata Levels in the Diet on Reproduction and Pre- Weaning Growth Performance of Guinea Pigs (Cavia porcellus L) Fed on Panicum maximum. International Journal of Animal Science and Technology, 2(4): 36-44. DOI: 10.11648/j.ijast.20180204.11

Mweugang NN, Tendonkeng F, Miégoué E, Matumuini FEN, Zougou TG, Fonteh FA, Boukila B, Pamo TE. 2016. Effets de l'inclusion de feuilles de manioc (Manihot esculenta Crantz) dans la ration sur les performances de reproduction du cobaye (Cavia porcellus L.) local camerounais. Int. J. Biol. Chem. Sci., 10(1): 269-280: DOI: http://dx.doi.org/10.4314/ijbcs.v10i1.21

Niraj CB, Vardhan HB. 2012. Impact of moringa leaves on erythrocytes maturation in a mammal Cavia porcellus. Indian Journal of Fundamental and Applied Life Sciences. An Online International Journal Available at http://www.cibtech.org/jls.htm, 2(2): 2629 /Niraj and Vardhan

Pamo TE, Boukila B, Fonteh FA, Tendonkeng F, Kana JR, Nanda AS. 2007. Nutritive values of some basic grasses and leguminous tree foliage of the Central region of Africa. Animal Feed Science and Technology, 135: 273-282.

Pamo TE, Niba AT, Fonteh FA, Tendonkeng F, Kana JR, Boukila B, Tsachoung. 2005. Effet de la supplémentation au Moringa oleifera ou au blocs multinutritionnels sur l'évolution du poids post-partum et la croissance pré-sevrage des cobayes (Cavia porcellus L.). Livestock Research for Rural Development, 17(4). http://www.lrrd.org/lrrd17/4/tedo17046. htm

Pauwels JM, Van Ranst E, Verloo M, Mvondo ze A. 1992. Méthode d'analyse de sols et de plantes, gestion de stock de verrerie et de produits chimiques. Manuel de Laboratoire de Pédologie. Publication Agricoles, 28.

Picron P. 2007. Amélioration de l'alimentation du cobaye en province de Kinshasa : Méthodes de prédiction de la valeur alimentaire des aliments. Mémoire de fin d'étude. UCL-AGRO, Louvain-laNeuve, 103 pp.

Steele RG, Torrie JH. 1980. Principles and Procedures of Statistics. McGraws Hill Book C: New York; 633 p.

Tolera A, Sundstøl F. 2000. Supplementation of graded levels of Desmodium intortum hay to sheep feeding on maize Stover harvested at three stages of maturity. 2 . Rumen fermentation and nitrogen metabolism. Animal Feed Science and Technology, 87: 215-229.

Zougou TG, Tendonkeng F, Miegoue E, Noumbissi MN, Mboko AV, Matumuini FEN, Boukila B, Pamo TE. 2017a. Performances de production des cobayes (Cavia porcellus L.) en fonction du niveau de protéines alimentaires. Int. J. Biol. Chem. Sci., 11(2): 828-840. DOI: https://dx.doi.org/10.4314/ijbcs.v11i2.24

Zougou TG, Tendonkeng F, Miégoué E, Noumbissi MNB, Matimuini NF, Mboko $\mathrm{AV}$, Lemoufouet J, Mweugang NN, Boukila B, Pamo ET. 2017b. Effet du niveau de protéines alimentaires sur la croissance post-sevrage et la carcasse chez le cobaye à l'Ouest Cameroun. Livestock Research for Rural Development, 29(5). http://www.lrrd.org/lrrd29/5/ften29105.h tml. 